\title{
Photoexcitation Circular Dichroism in Chiral Molecules
}

\author{
S. Beaulieu ${ }^{1,2}$, A. Comby ${ }^{1}$, D. Descamps ${ }^{1}$, B. Fabre ${ }^{1}$, G. A. Garcia ${ }^{3}$, R. Géneaux ${ }^{4}$, A. G. Harvey ${ }^{5}$, \\ F. Légaré ${ }^{2}$, Z. Mašín ${ }^{5}$, L. Nahon ${ }^{3}$, A. F. Ordonez ${ }^{5,6}$, S. Petit ${ }^{1}$, B. Pons ${ }^{1}$, Y. Mairesse ${ }^{1}$, O. \\ Smirnova $^{5,6}$, V. Blanchet ${ }^{1}$
}

${ }^{1}$ Université de Bordeaux - CNRS - CEA, CELIA, UMR5107, F33405 Talence, France

${ }^{2}$ Institut National de la Recherche Scientifique, Varennes, Quebec, Canada

${ }^{3}$ Synchrotron Soleil, l'orme des Merisiers, BP48, St Aubin, 91192 Gif sur Yvette, France

${ }^{4}$ LIDYL, CEA, CNRS, Université Paris-Saclay, CEA Saclay, 91191 Gif-sur-Yvette France.

${ }^{5}$ Max-Born-Institut, Max-Born-Str. 2A, 12489 Berlin, Germany

${ }^{6}$ Technische Universität Berlin, Ernst-Ruska-Gebäude, Hardenbergstr. 36 A, 10623, Berlin, Germany

Chirality is ubiquitous in nature and fundamental in science, from particle physics to metamaterials. The most established technique of chiral discrimination - photoabsorption circular dichroism - relies on the magnetic properties of a chiral medium and yields an extremely weak chiral response. We propose and demonstrate a new, orders of magnitude more sensitive type of circular dichroism in neutral molecules: photoexitation circular dichroism. It does not rely on weak magnetic effects, but takes advantage of the coherent helical motion of bound electrons excited by ultrashort circularly polarized light. It results in an ultrafast chiral response and the efficient excitation of a macroscopic chiral density in an initially isotropic ensemble of randomly oriented chiral molecules. We probe this excitation without 


\section{the aid of further chiral interactions using linearly polarized laser pulses. Our time-resolved study of vibronic chiral dynamics opens a way to the efficient initiation, control and moni- toring of chiral chemical change in neutral molecules at the level of electrons.}

The macro-world gives us many examples of chiral dynamics created by helical structures which convert rotations in a plane into translational motion orthogonal to it, from the Archimedes screw to plane propellers and household fans. In the micro-world, the electrons bound inside chiral molecules should develop a similar helical motion when excited by planar rotation of the electric field of circularly polarized light. Electronic excitation by circularly polarized light has been used to distinguish right-handed from left-handed molecules since $1896^{1}$. The technique, called the photoabsorption circular dichroism $(\mathrm{CD})^{2}$, is based on the difference in the absorption of leftand right-circularly polarized light in chiral molecules and remains the go-to tool ${ }^{3}$ for analysing properties of biological molecules, providing indispensable information on their structure, kinetics and thermodynamics, interaction with the environment and with other molecules. However, it does not rely on the helical nature of bound electron currents ${ }^{4}$, but uses the helical pitch of the light wave instead. This pitch, given by the wavelength of the absorbed light, $\lambda \gtrsim 2500 \AA\left(1 \AA=10^{-8}\right.$ $\mathrm{cm}$ ), is barely noticeable on the molecular scale of $\sim 1 \AA$, leading to very weak signals, three to four orders of magnitude less than the light absorption itself. Formally, the chiral-sensitive part of the light-induced excitation requires the excited electrons to respond to both the electric and the magnetic field of the light wave ${ }^{5}$, see Fig.(1a).

Remarkably, in spite of extraordinary recent advances in developing new methods for chiral 
discrimination that do not rely on the magnetic properties of the medium ${ }^{7-18}$, none has relied on detecting the helical motion of bound electrons. Is it possible to excite and probe such motion? We demonstrate both theoretically and experimentally that one can (i) induce chiral stereodynamics of bound electrons without the help of magnetic field effects, a new phenomenon we call PhotoeXcitation Circular Dichroism (PXCD); (ii) probe it with linearly polarized light without the help of the further chiral interactions that are usually presumed to be a prerequisite for chiral discrimination. Coherent excitation substitutes further chiral interactions at the probe step by coupling to a different quantum state of the same chiral molecule.

\section{Exciting chiral dynamics in bound states}

A hallmark of helical motion of bound electrons is the appearance of an induced dipole orthogonal to the polarization plane of the exciting circular light. We first show that an ultrashort pulse creates such a dipole in a randomly oriented molecular ensemble. Let the electric field of the pulse, rotating in the $x-y$ plane, coherently excites two states (Fig.1b) of a chiral molecule. As shown in the Supplementary Information (SI), the orientation-averaged induced dipole acquires the desired component along the light propagation direction $z$ :

$$
d_{z}^{P X C D} \propto \sigma\left[\vec{d}_{01} \times \vec{d}_{02}\right] \vec{d}_{12} \sin \left(\Delta E_{21} t\right)
$$

Here $\vec{d}_{01}, \vec{d}_{02}$ and $\vec{d}_{12}$ are the dipole transition vectors connecting the ground $|0\rangle$ and the two excited states $|1\rangle,|2\rangle$ (Fig. 1b), $\Delta E_{21}$ is the energy spacing between the excited states. For more than two states, Eq.(1) will contain the sum over all pairs of excited states $n, m$, leading to oscillations at all relevant frequencies $\Delta E_{n m}$. As a function of time the induced dipole vector maps out a helix (Fig. 
1b) and the z-component of the helical current is

$$
j_{z}^{P X C D} \propto \sigma\left[\vec{d}_{01} \times \vec{d}_{02}\right] \vec{d}_{12} \Delta E_{21} \cos \left(\Delta E_{21} t\right) .
$$

Both $d_{z}^{P X C D}$ and $j_{z}^{P X C D}$ are quintessential chiral observables (see e.g. ${ }^{19,20}$ ). Indeed, both are proportional to the light helicity $\sigma= \pm 1$ and to the triple product of three vectors $\left[\vec{d}_{01} \times \vec{d}_{02}\right] \vec{d}_{12}$. This product presents a fundamental measure of chirality: it changes sign upon reflection and thus has an opposite sign for left and right enantiomers. For randomly oriented non-chiral molecules $d_{z}^{P X C D}=j_{z}^{P X C D}=0$.

Eqs. $(1,2)$ lead to the following conclusions. First, the coherent excitation of electronic states leads to a charge displacement in the light propagation direction. Hence, a macroscopic dipole $d_{z}^{P X C D}$ and the corresponding chiral density are created in the excited states, with a chiral current oscillating out of phase for the two enantiomers. Second, PXCD requires no magnetic or quadrupole effects. Hence, it is orders of magnitude stronger than standard photoabsorption CD. While photoabsorption CD exploits the helical pitch of the laser field in space, PXCD takes advantage of the sub-cycle rotation of the light field in time and is inherently ultrafast. Indeed, PXCD arises only if the excitation dipoles $\vec{d}_{01}, \vec{d}_{02}$ are non-collinear: for the angle $\phi$ between the two transition dipoles, the PXCD (Eqs. $(1,2)$ ) is proportional to $\sigma \sin (\phi)$. Since $\sigma= \pm 1$, $\sigma \sin (\phi)=\sin (\sigma \phi)=\sin (\sigma \omega \tau)$, where $\omega$ is light frequency and $\tau=\phi / \omega$ is the required time for the light field to rotate by the angle $\phi$. PXCD vanishes if the coherence between excited states $|1\rangle$ and $|2\rangle$ is lost and reflects dynamical symmetry breaking in an isotropic medium.

The oscillations of the PXCD signal Eqs.(1,2) appear to suggest that probing it requires the 
combination of ultrafast time resolution and chiral sensitivity. We now show that time-resolving PXCD does not, in fact, require a chiral probe. The coherence underlying PXCD allows a chiral object to 'interact with itself', albeit in a different quantum state, thus mimicking interaction with "another chiral object" and removing any need for other chiral interactions during the probe step. One such non-chiral probe, termed PhotoeXcitation-induced photo-Electron Circular Dichroism (PXECD), is introduced below.

\section{Probing chiral dynamics in bound states}

One way to probe the excited chiral density is to promote the chiral wave-packet to the electron continuum using a linearly polarized pulse (Fig 1c). As shown in the SI, the standard photoionization observable, the photoelectron current averaged over molecular orientations, is:

$$
J_{z}^{P X E C D}(k)=\sigma\left[\vec{d}_{01} \times \vec{d}_{02}\right] \vec{D}_{12}^{r}(k) \sin \left(\Delta E_{21} \tau\right)-\sigma\left[\vec{d}_{01} \times \vec{d}_{02}\right] \vec{D}_{12}^{i}(k) \cos \left(\Delta E_{21} \tau\right),
$$

with $J_{x}^{P X E C D}(k)=J_{y}^{P X E C D}(k)=0$. Here $\tau$ is the pump-probe delay, $\vec{D}_{12}(k)=\vec{D}_{12}^{r}(k)+$ $i \vec{D}_{12}^{i}(k)$ is the Raman-type photoionization vector (see the SI) which connects the excited bound states via the common continuum and plays the role of $\vec{d}_{12}$ of Eq.(1,2) and $k$ is the photoelectron momentum.

First, the electron current Eq. (3) is proportional to the helicity $\sigma$ of the pump pulse. Second, as transitions to the continuum are described by complex dipole vectors, it contains two triple

products. Just like the triple product $\left[\vec{d}_{01} \times \vec{d}_{02}\right] \vec{d}_{12}$ earlier, both $\left[\vec{d}_{01} \times \vec{d}_{02}\right] \vec{D}_{12}^{r}$ and $\left[\vec{d}_{01} \times \vec{d}_{02}\right] \vec{D}_{12}^{i}$ will change sign upon reflection. Thus, the electron current reverses its direction if the handedness 
of the pump pulse or of the enantiomer is swapped, showing that PXECD is a genuine chiral effect. The chiral nature of the response arises only if the participating bound states are coherently excited. Once the coherence is lost, the chiral signal will also disappear.

Importantly, the state of the continuum (Fig 1c) does not need to be chiral, as it only provides a link between the two chiral bound states. $J_{z}^{P X E C D}(k)$ remains chiral even for a plane wave continuum (see the SI), in this case $\vec{D}_{12}(k)$ only has an imaginary component:

$$
J_{z, P W}^{P X E C D}(k)=-\sigma\left[\vec{d}_{01} \times \vec{d}_{02}\right] \vec{D}_{12}^{i, P W}(k) \cos \left(\Delta E_{21} \tau\right)
$$

The total photoelectron current $J_{t o t}^{P X C D}=\int J_{z, P W}^{P X E C D}(k) d k$ measures the helical current excited in bound states $j_{z}^{P X C D}$ (Eq. 2) distorted by the partial alignment of the molecular ensemble induced by the pump (see the SI). One might think that partial alignment of the excited molecular ensemble could already be fully responsible for enabling non-chiral probes of chiral dynamics. It is not true in our case. Indeed, the effect of alignment persists for a single excited electronic state and for the two excited electronic states with collinear dipoles, but in both cases it leads to zero PXECD current. Finally, removing the effect of partial alignment from Eq.(4) shows that the PXECD current remains chiral for every $k$, while $J_{t o t}^{P X C D}$ becomes directly proportional to the chiral component of the helical current in bound states: $J_{t o t}^{P X C D} \propto j_{z}^{P X C D}$ (see the SI).

Probing the created chiral excitation using photo-electron imaging with linearly polarized light constitutes yet another new phenomenon, PhotoeXcitation-induced photoElectron Circular Dichroism (PXECD). PXECD is reminiscent of the Photoelectron Circular Dichroism (PECD) $7-9,16-18,21$, which arises when a circularly polarized light is used to photoionize a chiral molecule. 
However, there is a fundamental difference. PECD can only exist if the molecular potential felt by the emitted electron is chiral ${ }^{7}$ (the effect becoming negligible for photoelectron energies above 10 $\mathrm{eV}$ ), while the initial orbital may or may not be chiral at all ${ }^{22}$. It is also clear from the diagram of PECD in Fig 1 (d). The continuum state cannot merely serve as a non-chiral link, as in this case it will only mediate the coupling of the chiral object, the molecule in the ground state, "to itself" rather than to another chiral object.

In contrast to PECD, the PXECD requires neither chiral light, nor chiral scattering of the photo-electron. Since PXECD does not require the technically challenging use of ultrashort circularly polarized XUV pulses ${ }^{23-27}$, it opens unique perspectives for ultrafast chiral-sensitive measurements using readily available linearly polarized UV and XUV light from table-top high harmonic generation sources, with no restrictions on photoelectron energies.

We shall now confirm both numerically and experimentally that our scheme provides a sensitive time-resolved probe of chiral molecular dynamics in bound states.

\section{Theoretical analysis in fenchone}

To quantify the PXECD effect we performed quantum mechanical calculations on fenchone molecules (see the SI). First, we simulated the PXCD phenomenon and calculated the excitation of the s- and p-manifold of Rydberg states in fenchone by a circular pump pulse. The resulting electron density of the Rydberg wave-packet is asymmetric in the $z$-direction in the momentum space. The asymmetry reverses if the helicity of the pump pulse or the handedness of the molecule is reversed. The 
strength of the PXCD can be quantified by the magnitude of the chiral component of the excited electron density. It is obtained by subtracting the momentum space density $D$ obtained with right (R) and left (L) polarized light: $P X C D=2(D(L)-D(R)) /(D(L)+D(R))$. Even after averaging over molecular orientations, the calculated PXCD reaches very high values $(35 \%$, Fig. $2(\mathrm{a}))$. The

asymmetry of the charge distribution corresponds to a macroscopic dipole moment $d_{z}^{\mathrm{PXCD}}$ which reaches 3 Debye (Fig. 2(b)) and oscillates at frequencies determined by the energy differences between the states forming the electronic wave-packet. The calculated pump-probe PXECD signal reveals these oscillations (Fig. 2c). While few-femtosecond pulses would be needed to resolve them, the PXECD signal can also be detected with much longer pulses. Fig. 2(d) shows that both PXCD and PXECD survive temporal averaging over $100 \mathrm{fs}$ duration of a probe pulse.

\section{Observation of PXECD in fenchone}

In our experiment, a circularly polarized femtosecond pump pulse at $201 \mathrm{~nm}(6.17 \mathrm{eV}$ photon energy, $80 \mathrm{meV}$ at 1/e bandwidth) photoexcites enantiopure fenchone molecules from a supersonic gas jet in the interaction zone of a velocity map imaging spectrometer. The molecules are excited to their first ( $s$ - and $p$-) Rydberg bands through single-photon absorption (Fig. 3 (a), see the SI). A time-delayed, linearly polarized probe pulse at $405 \mathrm{~nm}$ (3.1 eV photon energy, $35 \mathrm{meV}$ FWHM bandwidth) induces one-photon ionization of the excited molecules. The cross-correlation of the pump and the probe pulses is $170 \mathrm{fs}$. The photoelectrons are accelerated and projected by an electrostatic lens onto a set of dual microchannel plates and imaged by a phosphor screen and a CCD camera. The photoelectron images are recorded alternatively using left (LCP) and right 
(RCP) circularly polarized pump pulses. The difference (LCP-RCP) and sum (LCP+RCP) of these two images are reconstructed using a least-square fitting algorithm (see the SI). We define the PXECD signal as $P X E C D=\frac{2(L C P-R C P)}{(L C P+R C P)}$ and the photoelectron spectrum (PES) as $P E S=$ $(L C P+R C P) / 2$. Both are shown in Fig. 3(b) for a 200 fs pump-probe delay. As expected, a significant PXECD signal is observed, reaching $1 \%{ }^{28}$ in good agreement with our calculations (Fig. 2(d)).

The photoelectron spectrum contains a single broad component, corresponding to ionization from the outermost orbital (vertical ionization potential $8.72 \mathrm{eV}$ ). The position of this component does not shift with the pump-probe delay (Fig. 4 (b)) and decays in 3.3 ps, reflecting simple vibronic relaxation of the Rydberg population onto lower states which cannot be photoionized by the $3.1 \mathrm{eV}$ probe photons. The temporal evolution of the PXECD image shows much richer spectroscopic features, which can be analyzed by decomposing it in odd Legendre polynomials (Fig. 4(a)). We note that a sum of first- and third-order Legendre polynomials, with coefficients $\alpha$ and $\alpha^{\prime}$, is enough to get the PXECD images. Both coefficients maximize around $\sim 50$ meV below the maximum of the PES. The PXECD signal (Fig. 4(b)) can be decomposed into two components: below and above the maximum of the PES. The low-energy component of $\alpha$ undergoes a rather smooth decay. On the contrary, its high-energy component decays very quickly and even changes sign around 1 ps. For $\alpha^{\prime}$ the behaviour is opposite, i.e. the high-energy component shows much slower dynamics than the low-energy part. Such time- and electron energy- dependent behaviour is characteristic of internal vibrational torsional motion and may indicate the change of the chiral structure of the molecule induced by such motion. Indeed, the electronic excitation of the 
molecules is expected to be accompanied by a significant vibrational excitation, since the equilibrium geometries of the $3 \mathrm{~s}$ and $3 \mathrm{p}$ Rydberg states are quite different from that of the ground state. The molecules will tend to relax towards the equilibrium geometry of the Rydberg states, and oscillate around it. Figure 5 illustrates the influence of this change of molecular geometry on the calculated PXECD signal. Even small bond length changes $(\leq 7 \%)$ lead to significant modification of the PXECD signal. This demonstrates the remarkable sensitivity of PXECD to molecular vibrations, which follow the electronic excitation. At 4 ps (not shown), the PXECD completely vanishes while the Rydberg population is still significant. This result unambiguously reflects the loss of wave-packet coherence which halts chiral dynamics in our experiment.

\section{Vibrational PXCD: experiments in camphor}

Is it possible to create PXCD from purely vibrational excitation of a chiral molecule? Theoretically, the two excited states in Eqs.(1,2) needed for PXCD do not have to be different electronic states. Vibrational states within the same electronic state can also fulfil the PXCD condition as long as their dipoles are not collinear, see Eqs. $(1,2)$. As shown in the SI, this requires the breakdown of the Franck-Condon approximation, which is caused by a strong dependence of the electronic wave-function on the position of the nuclei. In turn, such dependence leads to the appearance of electronic currents stimulated by the nuclear motion, which is triggered by the pump pulse. Thus, vibrational PXCD is intertwined with the underlying chiral motion of electrons. Note that this strong dependence of the electronic wave-functions on the nuclear positions naturally arises in

the vicinity of conical intersections between electronic potential surfaces. Thus, we expect that 
PXECD could be used to excite and reveal coherent chiral dynamics at conical intersections.

To gain further insight into the role of electronic versus vibrational dynamics in PXECD, we performed measurements in (1R)-(+)-camphor, a very similar structural isomer of fenchone. The $s$ - and $p$ - Rydberg bands of camphor are upshifted by additional several tens of meV compared to fenchone, preventing direct excitation of the $p$ - states and thus of an electronic chiral wavepacket. Nevertheless, the experiment still reveals a strong PXECD signal, indicating that a chiral vibronic wave-packet has been created in the s- Rydberg band of camphor. The $\alpha^{\prime}$ coefficients in camphor and fenchone are of opposite sign as seen in multiphoton ${ }^{29}$ and one-photon PECD ${ }^{17}$. In our experiment, this could be a consequence of PXECD sensitivity to isomerism (see Figure 5 to gauge the sensitivity to nuclear configuration), but it could also be a signature of the different nature of the excited chiral electronic currents in fenchone and camphor. Changing the excitation wavelength from $202 \mathrm{~nm}$ to $200 \mathrm{~nm}$ does not affect the monoexponential decay of the PES. In contrast, a strong change is observed in the PXECD: the $\alpha^{\prime}$ magnitude is almost twice as large and it is shifted in energy towards the red wing of the photoelectron spectrum. The drastic change observed in the PXECD signal in camphor once the pump photon energy is increased by only 60 $\mathrm{meV}$ illustrates the extreme sensitivity of this measurement to the excited vibrational dynamics.

\section{Conclusions and outlook}

We have demonstrated two new phenomena. First, we have shown the efficient excitation of a macroscopic bound chiral electron density in the excited states of randomly oriented chiral 
molecules without the help of magnetic interactions (the PXCD phenomenon). In the dipole approximation the chiral pitch of circularly polarized light vanishes. This means that the creation of the macroscopic chiral density in the isotropic ensemble of chiral molecules is based not on the helical structure of light, but on its planar rotation.

Second, we have shown that the resulting chiral dynamics can be probed without the help of further chiral interactions and thus in an efficient and versatile way. The detection relies on photoelectron circular dichroism arising from the ionization of excited molecules by linearly polarized light pulses (the PXECD phenomenon), but is not limited to this scheme. The application of a linearly polarized XUV probe in PXECD would enable genuine probing of ultrafast chiral bound dynamics, since PXECD does not require chiral interaction in the continuum, which becomes negligible for sufficiently high-energy electrons.

The ensemble-averaged chiral charge density arising in PXCD implies asymmetry in charge distribution along the light propagation direction. Depending on the medium density, this could lead to a very large coherently oscillating macroscopic dipole. The phase of this oscillation is opposite for two enantiomers, leading to macroscopic enantio-sensitive effects. The existence of the enantio-sensitive macroscopic dipole opens the way to the separation of enantiomers in isotropic racemic mixtures in the gas phase.

The PXCD phenomenon opens the way to direct visualization of chiral electronic density using time-resolved X-ray diffraction imaging, both in the gas and condensed phase. Intense ultrafast sources of X-ray radiation, such as Free Electron Lasers, combined with measurements, sensitive 
to valence-shell dynamics in the gas phase ${ }^{30}$ should lead to few-fs time resolution of chiral charge dynamics.

Finally, PXCD could be used to drive molecular reactions in chiral systems in a stereospecific way, by imprinting a chiral torque via the helicity of the exciting circularly polarized pulse. The ultrafast charge dynamics triggered by coherent electronic excitation is reminiscent of ultrafast charge migration triggered by photo-ionization ${ }^{31-34,36-38}$ recently observed in ref. ${ }^{39}$ and speculated to underlie charge-directed reactivity in cations ${ }^{35}$. Chiral electron stereo-dynamics in neutral molecules may open similar opportunities for controlling charge and energy flow in molecules at the level of electrons, offering new perspectives for such intriguing problems as asymmetric synthesis, a major challenge in stereochemistry.

\section{Methods}

An Even-Lavie valve is used as a pulsed enantiopure fenchone source with helium as carrier gas to avoid cluster formation. (1R)-(-) and (1S)-(+)-fenchone correspond to $(1 \mathrm{R}, 4 \mathrm{~S})$ and $(1 \mathrm{~S}, 4 \mathrm{R})$ fenchone respectively. The $170 \mathrm{fs}$ cross-correlation time as well as the $0 \mathrm{fs}$ delay are determined on the lightest fragment $\mathrm{C}_{4} \mathrm{H}_{5} \mathrm{O}^{+}$produced by dissociative ionization with both linearly polarized pump and probe. The high voltage of the repeller electrode was $-3 \mathrm{kV}$ for the experiment done in fenchone and only $-2 \mathrm{kV}$ for the experiment done in Camphor, which increases the energy resolution. Note that the latter, along with the energy calibration, that has been determined by photoionizing krypton. Typically the energy resolution is $80 \mathrm{meV}$ at $0.7 \mathrm{eV}$ kinetic energy. The presented results are obtained by scanning the pump-probe delays typically 30 times. At each delay, helicity is 
permuted each 45000 laser shots (=45 seconds) to record several images.

1. Cotton, A. Recherches sur l'absorption et la dispersion de la lumire par les milieux doués du pouvoir rotatoire. J. Phys. Theor. Appl. 5, 237-244 (1896).

2. Barron, L. D. Molecular light scattering and optical activity (Cambridge University Press, Cambridge, UK ; New York, 2004), 2nd ed., rev. and enl edn.

3. Berova, N., Nakanishi, K. \& Woody, R. Circular dichroism: principles and applications (Wiley-VCH, New York, 2000), 2nd ed edn.

4. For non-magnetic materials, light absorption is proportional to the scalar product of the induced polarization current and electric component of the light field. Thus, even if the motion of bound electrons underlying the induced current were helical, moving out of the polarization plane of the circular light, it would have not contributed to the CD signal. For magnetic materials light absorption includes an additional term: a scalar product between magnetic field vector and the time-derivative of induced magnetization. It does not change our conclusion: CD is only sensitive to electron dynamics in light polarization plane also for magnetic materials.

5. Electric quadrupole effects vanish in isotropic ensembles of chiral molecules ${ }^{6}$.

6. Craig, D. P. \& Thirunamachandran, T. Molecular quantum electrodynamics: an introduction to radiation-molecule interactions (Courier Corporation, 1984).

7. Ritchie, B. Theory of the angular distribution of photoelectrons ejected from optically active molecules and molecular negative ions. Phys. Rev. A 13, 1411-1415 (1976). 
8. Powis, I. Photoelectron circular dichroism of the randomly oriented chiral molecules glyceraldehyde and lactic acid. The Journal of Chemical Physics 112, 301-310 (2000). URL http://dx.doi.org/10.1063/1.480581.

9. Bowering, N. et al. Asymmetry in Photoelectron Emission from Chiral Molecules Induced by Circularly Polarized Light. Physical Review Letters 86, 1187-1190 (2001). URL http://dx.doi.org/10.1103/PhysRevLett.86.1187.

10. Lux, C. et al. Circular dichroism in the photoelectron angular distributions of camphor and fenchone from multiphoton ionization with femtosecond laser pulses. Angewandte Chemie International Edition 51, 5001-5005 (2012). URL http://dx.doi.org/10.1002/anie.201109035.

11. Lehmann, C. S., Ram, N. B., Powis, I. \& Janssen, M. H. M. Imaging photoelectron circular dichroism of chiral molecules by femtosecond multiphoton coincidence detection. The Journal of Chemical Physics 139, 234307 (2013). URL http://dx.doi.org/10.1063/1.4844295.

12. Pitzer, M. et al. Direct determination of absolute molecular stereochemistry in gas phase by coulomb explosion imaging. Science 341, 1096-1100 (2013). URL http://dx.doi.org/10.1126/science.1240362.

13. Herwig, P. et al. Imaging the absolute configuration of a chiral epoxide in the gas phase. Science 342, 1084-1086 (2013). URL http://dx.doi.org/10.1126/science.1246549. 
14. Patterson, D., Schnell, M. \& Doyle, J. M. Enantiomer-specific detection of chiral molecules via microwave spectroscopy. Nature 497, 475-477 (2013). URL http://dx.doi.org/10.1038/nature12150.

15. Yachmenev, A. \& Yurchenko, S. N. Detecting Chirality in Molecules by Linearly Polarized Laser Fields. Physical Review Letters 117 (2016). URL http://dx.doi.org/10.1103/PhysRevLett.117.033001.

16. Comby, A. et al. Relaxation Dynamics in Photoexcited Chiral Molecules Studied by Time-Resolved Photoelectron Circular Dichroism: Toward Chiral Femtochemistry. The Journal of Physical Chemistry Letters 7, 4514-4519 (2016). URL http://dx.doi.org/10.1021/acs.jpclett.6b02065.

17. Nahon, L., Garcia, G. A. \& Powis, I. Valence shell one-photon photoelectron circular dichroism in chiral systems. Journal of Electron Spectroscopy and Related Phenomena 204, 322 (2015). URL http://dx.doi.org/10.1016/j.elspec.2015.04.008.

18. Nahon, L. et al. Determination of accurate electron chiral asymmetries in fenchone and camphor in the vuv range: Sensitivity to isomerism and enantiomeric purity. Phys. Chem. Chem. Phys. 18, 12696-706 (2016). URL http: / / dx . doi.org/10.1039/C6CP01293K.

19. Barron, L. D. True and false chirality and absolute asymmetric synthesis. Journal of the American Chemical Society 108, 5539-5542 (1986). URL http://dx.doi.org/10.1021/ja00278a029. 
20. Tang, Y. \& Cohen, A. E. Optical chirality and its interaction with matter. Physical Review Letters 104163901 (2010). URL http://dx.doi.org/10.1103/PhysRevLett.104.163901.

21. Garcia, G. A., Nahon, L., Daly, S. \& Powis, I. Vibrationally induced inversion of photoelectron forward-backward asymmetry in chiral molecule photoionization by circularly polarized light. Nature Communications 4 (2013). URL http://dx.doi.org/10.1038/ncomms3132.

22. Ulrich, V. et al. Giant chiral asymmetry in the c 1 s core level photoemission from randomly oriented fenchone enantiomers. The Journal of Physical Chemistry A 112, 3544-3549 (2008).

23. Wang, T. et al. Femtosecond single-shot imaging of nanoscale ferromagnetic order in $\mathrm{Co} / \mathrm{Pd}$ multilayers using resonant x-ray holography.. Phys. Rev. Lett. 108, 267403 (2012) URL http://dx.doi.org/10.1103/PhysRevLett.108.267403.

24. Spezzani, C. et al. Coherent light with tunable polarization from singlepass free-electron lasers.. Phys. Rev. Lett. 107, 084801 (2011) URL http://dx.doi.org/10.1103/PhysRevLett.107.084801.

25. Allaria, E. et al., Highly coherent and stable pulses from the FERMI seeded freeelectron laser in the extreme ultraviolet.. Nat. Photon. 6, 699704 (2012) URL http://dx.doi.org/10.1038/nphoton.2012.233. 
26. Fleischer, A. et al., Spin angular momentum and tunable polarization in high-harmonic generation. Nat. Photon. 8, 543-549 (2014) URL http://dx.doi.org/10.1038/nphoton.2014.108.

27. Ferré, A. et al. A table-top ultrashort light source in the extreme ultraviolet for circular dichroism experiments. Nature Photonics 9, 93-98 (2015). URL http://dx.doi.org/10.1038/nphoton.2014.314.

28. The Forward-Backward Asymmetry, i.e. the normalized difference of photoelectron counts in forward and backward hemispheres relative to $\mathrm{z}$ axis, is $0.62 \% \pm 0.03 \%$.

29. Lux, C., Wollenhaupt, M., Sarpe, C. \& Baumert, T. Photoelectron circular dichroism of bicyclic ketones from multiphoton ionization with femtosecond laser pulses. ChemPhysChem 16, 115-137 (2015). URL http://dx.doi.org/10.1002/cphc.201402643.

30. Bredtmann, T., Ivanov, M. \& Dixit, G. X-ray imaging of chemically active valence electrons during a pericyclic reaction. Nat Commun 5 (2014).

31. Lunnemann, S. et al. Ultrafast charge migration in 2-phenylethyl-N, N-dimethylamine. Chem. Phys. Lett. 450450 (2008).

32. Breidbach, J. et al. Universal attosecond response to the removal of an electron. Phys. Rev. Lett. 94033901 (2005).

33. Remacle, F. et al. An electronic time scale in chemistry. Proc. Natl. Acad. Sci. USA 1036793 (2006). 
34. Kuleff, A. I. et al. Charge migration in different conformers of glycine: The role of nuclear geometry. Chem. Phys. 3386793 (2007).

35. Weinkauf, R. et al. Nonstationary electronic states and site-selective reactivity. J. Phys. Chem. A 1017702 (1997).

36. Lepine, F. et al. Attosecond molecular dynamics: Fact or fiction?. Nat. Phot. 8195204 (2014).

37. Leone, S. R. et al. What will it take to observe processes in 'real time'?. Nat. Phot. 8 162-166 (2014).

38. Kuleff, A. I. et al. Ultrafast correlation-driven electron dynamics. J. Phys. B 47124002 (2014).

39. Calegari, F. et al. Ultrafast electron dynamics in phenylalanine initiated by attosecond pulses. Science 346 336-339 (2014).

40. Pulm, F., Schramm, J., Hormes, J., Grimme, S. \& Peyerimhoff, S. D. Theoretical and experimental investigations of the electronic circular dichroism and absorption spectra of bicyclic ketones. Chemical Physics 224, 143-155 (1997). URL http://dx.doi.org/10.1016/S0301-0104(97)00258-9. 


\section{Acknowledgements}

We thank Rodrigue Bouillaud and Laurent Merzeau for technical assistance. We thank M. Ivanov, A. Stolow and T. Elsaesser for stimulating discussions. We acknowledge financial support of the Agence Nationale pour la Recherche (ANR-14-CE32-0014 MISFITS) and the University of Bordeaux. Z.M. and O.S. gratefully acknowledge the support from Deutsche Forschungsgemeinschaft, project Sm 292-5/1, A.H. gratefully acknowledges the support from Deutsche Forschungsgemeinschaft, project Iv 152/7-1. A.F.O. and O.S. gratefully acknowledge EU ITN MEDEA -AMD641789-17 project. S.B. acknowledges the support of a NSERC Vanier Canada Graduate Scholarship. R.G. acknowledges financial support from the Agence Nationale pour la Recherche through the XSTASE project (ANR-14-CE32-0010).

\section{Additional information}

Competing Interests The authors declare that they have no competing financial interests. Requests for materials and correspondence should be addressed to valerie.blanchet@celia.u-bordeaux.fr, yann.mairesse@celia.u-bordeaux.fr, Olga.Smirnova@mbi-berlin.de, bernard.pons@u-bordeaux.fr

\section{Author contributions}

S.B., A.C., R.G., Y.M., V.B., performed the experiment. D.D. and S.P. operated the laser system. S.B., A.C., B.F., G.G., L.N., B.P., Y.M. and V.B. analyzed the data. B.F. and B.P. performed the molecular geometry and dynamical calculations. A.H., Z.M. and O.S. developed the analytical 
theory, A.F.O. and O.S. derived chirality measures for PXCD and PXECD. S.B. wrote the first version of the manuscript, all authors contributed to writing the manuscript. 
FIGURE 1: Chiral discrimination schemes. C.C. denotes complex conjugated, i.e. timereversed process. Downarrows denote C.C. of driving fields. (a) CD requires magnetic dipole transition up and electric dipole transition down and vise-versa. (b) PXCD (Eq.1) requires coherent excitation of two states by ultrashort circularly polarized pulse. The stimulated dipole transition to state $\mid 2>$ is followed by dipole transition to state $\mid 1>$ and stimulated dipole transition to state $\mid 0$ >. Insert: Induced dipole maps out a helix as a function of time. (c) In PXECD (Eq.3) the two excited states are connected by Raman-type transitions via continuum, stimulated by linearly polarized pulse. (d) PECD requires circularly polarized light and photoelectron scattering off chiral potential $V_{c h}$.

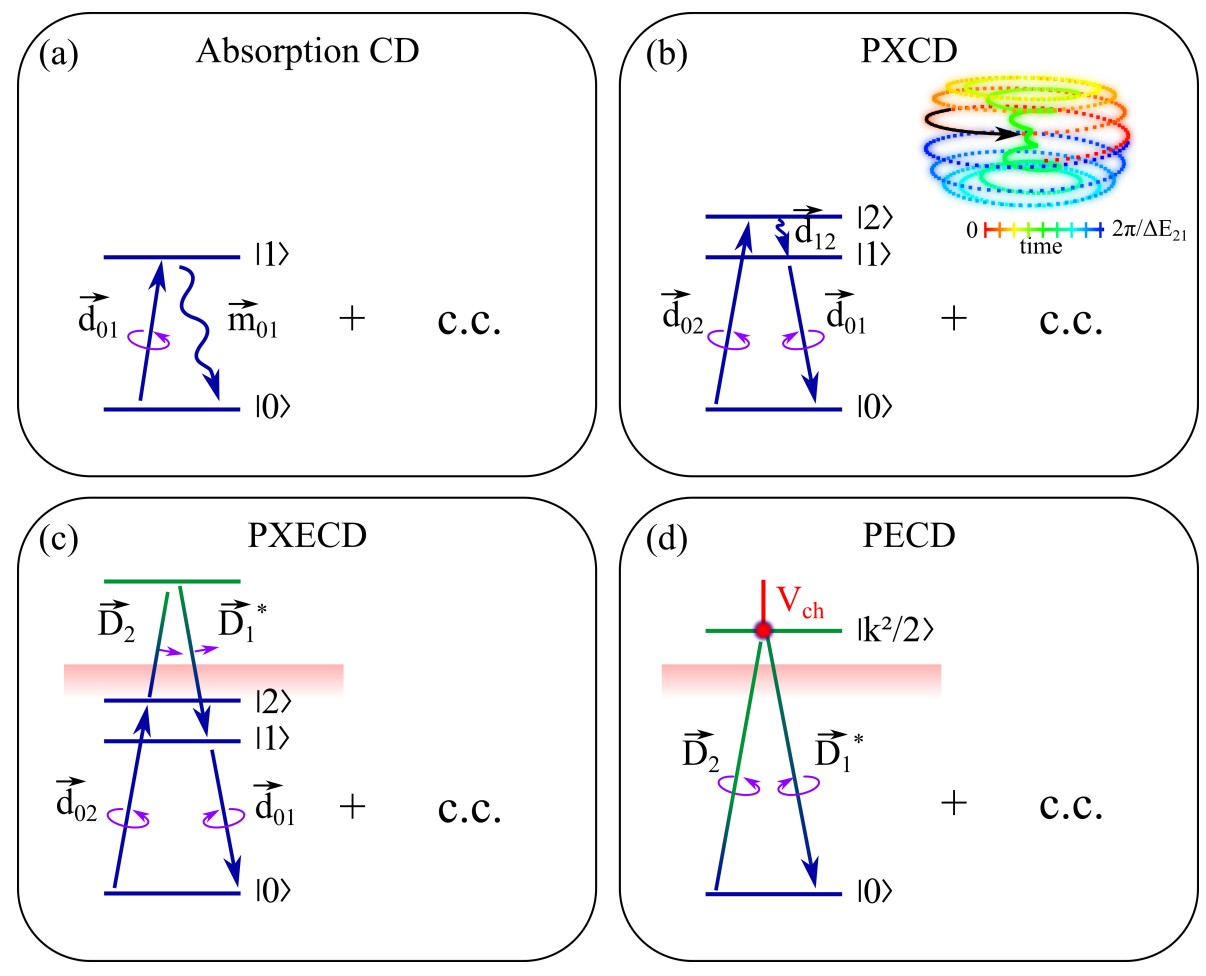


FIGURE 2: Theoretical analysis of electron chiral dynamics in (1S)-(+)-fenchone. (a) Momentum space electron density underlying PXCD. The asymmetry signifying chirality of the electron density is formed in light propagation direction z. (b) Temporal evolution of the $x^{-}, y_{-}$and z-components of the macroscopic dipole associated with the (3s,3p) Rydberg wave-packet created by a pump pulse (shaded area). Only the z-component, along the direction of propagation of the pump and probe pulses, survives orientational averaging. (c) Momentum space PXECD signals at various pump-probe delays $t$. (d) Time-averaged PXECD to account for the temporal resolution of the experiment.

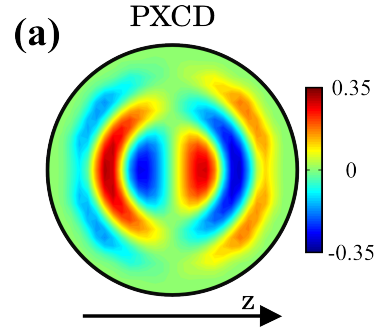

(b)

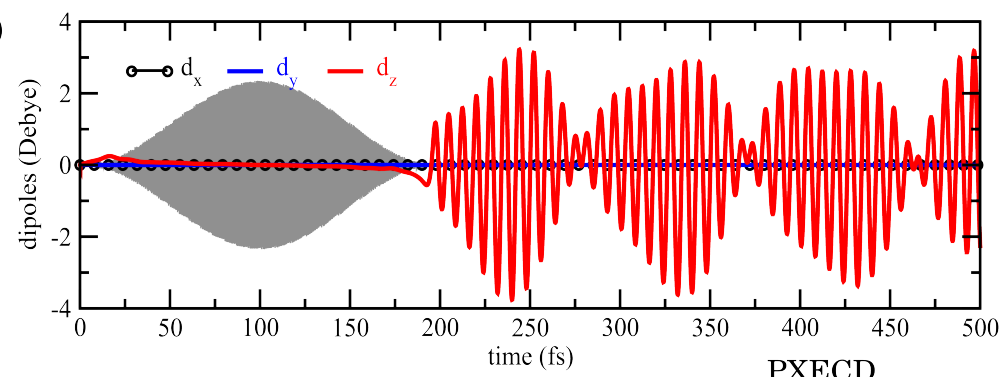

(c)

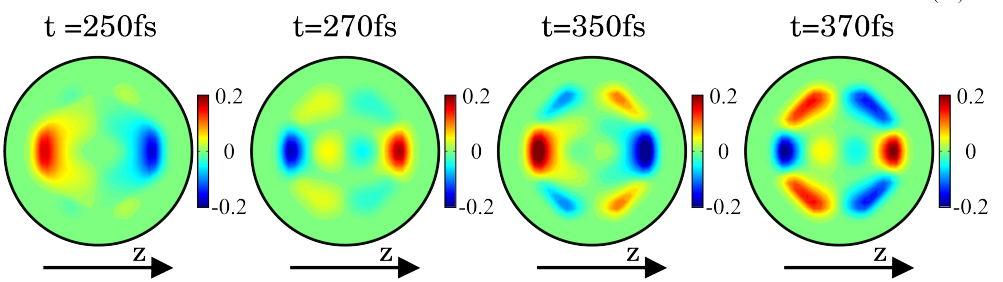

(d)

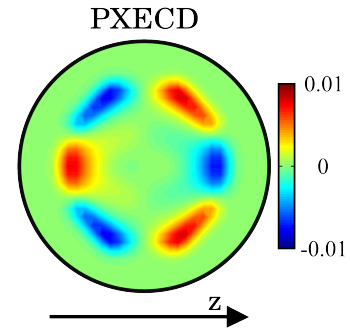


FIGURE 3 : PXECD in fenchone molecules. (a) Absorption of circularly polarized pulse at $201 \mathrm{~nm}$ with helicity $\sigma= \pm 1$ promotes an electron from the highest occupied molecular orbital to the $s$ - and $p$ - Rydberg bands, creating a chiral electron wave-packet. A linear probe pulse at 405 $\mathrm{nm}$ photoionizes the molecule, revealing the chiral asymmetry of the Rydberg wave-packet in the angular distribution of the photoelectrons. The absorption spectrum of fenchone is adapted from 40 (b) Experimental image of photoelectron spectrum (PES) and PXECD images at 200 fs pumpprobe delay for (1S)-(+)-fenchone. The characteristic forward-backward asymmetry is observed in light propagation direction $\mathrm{z}$.

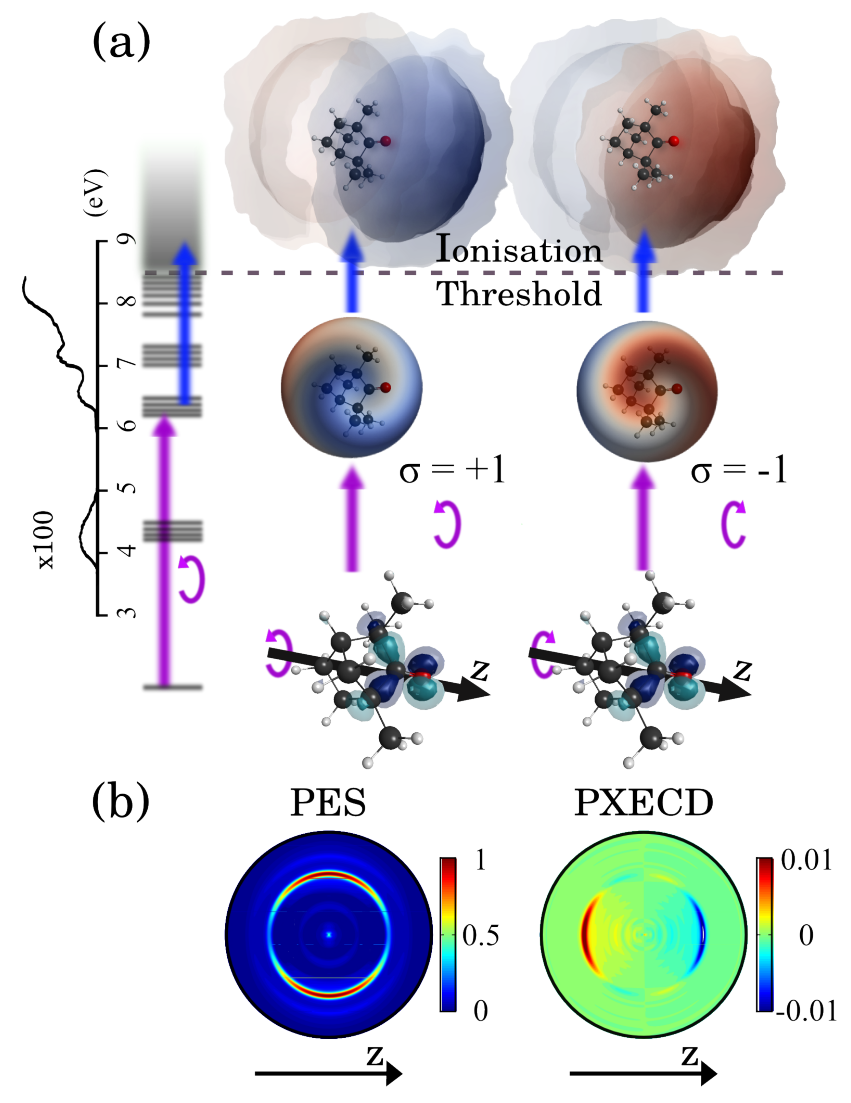


FIGURE 4: Time-Resolved PXECD in fenchone and camphor. (a) Legendre polynomials decomposition of the PXECD image for (1S)-(+)-Fenchone at $200 \mathrm{fs}$ pump-probe delay. The $\alpha, \alpha^{\prime}$ coefficients are multiplied by their associated Legendre polynomials $P_{i}(\theta): P_{1}=\cos (\theta)$, $P_{3}=\left(5 / 2 \cdot \cos ^{3}(\theta)+3 / 2 \cdot \cos (\theta)\right)$. (b) Evolution of the PES and PXECD coefficients as a function of pump-probe delay, in (1S)-(+)-fenchone with $201 \mathrm{~nm}$ pump, (1R)-(+)-camphor with 202 $\mathrm{nm}$ pump and with $200 \mathrm{~nm}$ pump. The black dotted lines represent energies corresponding to the maximum of PES. $\alpha$ and $\alpha^{\prime}$ are normalized to the maximum value of PES.

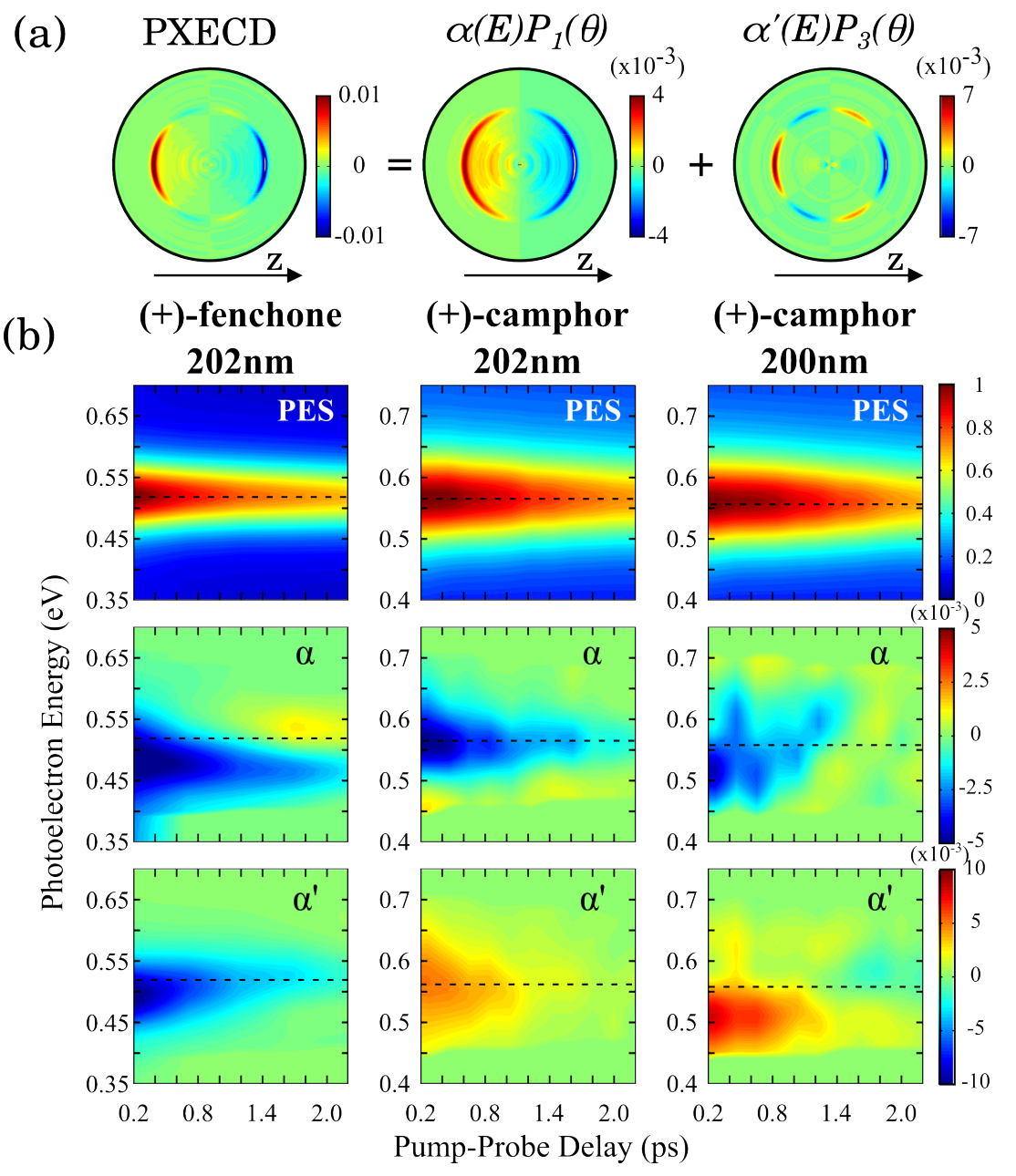


FIGURE 5: Sensitivity of PXECD in (1S)-(+)-fenchone to the evolution of the chiral molecular structure. (a) The equilibrium geometries of the ground (dark) and 3s (shadowed) electronic states, and the PXECD signal computed at the ground state geometry for both pump excitation and probe ionization. (b) The representation of the geometries are exchanged and the PXECD is computed assuming that pump excitation occurs at the ground state geometry while probe ionization occurs once the vibronic wave-packet has reached the 3s equilibrium geometry. The PXECD image is averaged over random molecular orientations and the $100 \mathrm{fs}$ duration of the probe pulse.

(a)
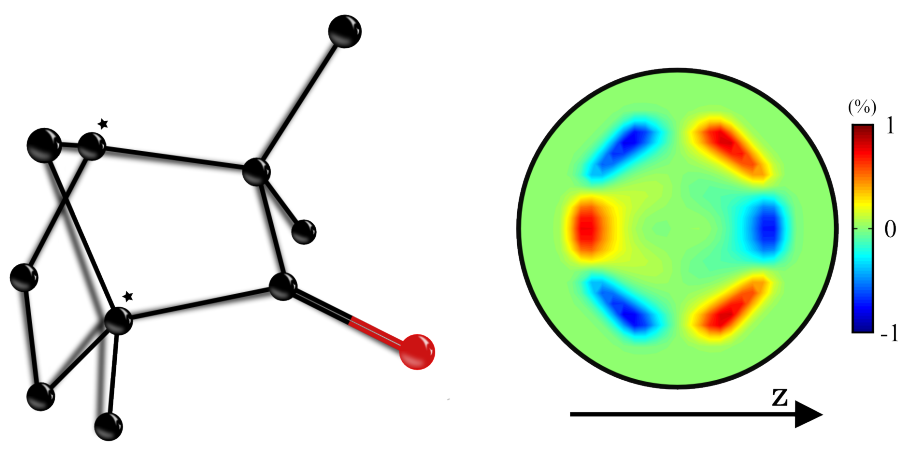

(b)
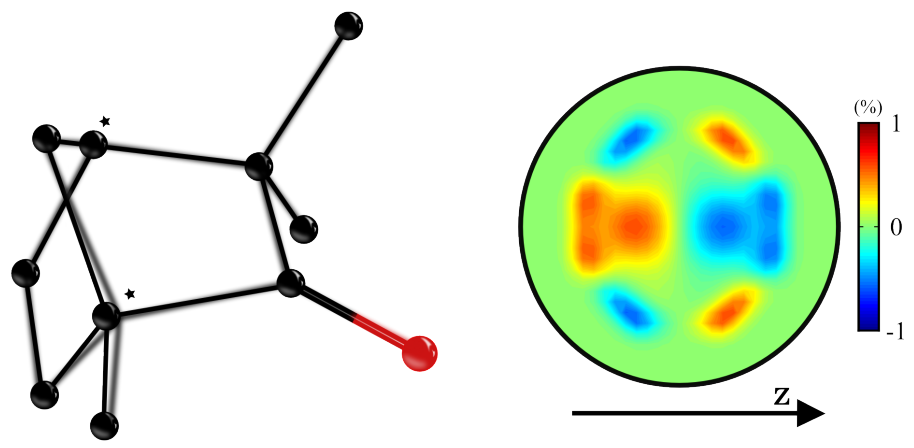